\title{
Chlorotoxin-conjugated graphene oxide for targeted delivery of an anticancer drug
}

\author{
This article was published in the following Dove Press journal: \\ International Journal of Nanomedicine \\ 18 March 2014 \\ Number of times this article has been viewed
}

\section{Hao Wang' \\ Wei $\mathrm{Gu}^{2}$ \\ Ning $\mathrm{Xiao}^{2}$ \\ Ling $\mathrm{Ye}^{2}$ \\ Qunyuan Xu'}

'Regeneration and Repair, Key Laboratory for Neurodegenerative Disease of The Ministry of Education, ${ }^{2} \mathrm{School}$ of Chemical Biology and Pharmaceutical Sciences, Capital Medical University, Beijing, People's Republic of China
Correspondence: Qunyuan Xu Regeneration and Repair, Key Laboratory for Neurodegenerative Disease of The Ministry of Education, Capital Medical University, Beijing, People's Republic of China

Tel +86 I0 839| |464

Fax +861083911464

Email xuqy@ccmu.edu.cn
Abstract: Current chemotherapy for glioma is rarely satisfactory due to low therapeutic efficiency and systemic side effects. We have developed a glioma-targeted drug delivery system based on graphene oxide. Targeted peptide chlorotoxin-conjugated graphene oxide (CTX-GO) sheets were successfully synthesized and characterized. Doxorubicin was loaded onto CTX-GO (CTX-GO/DOX) with high efficiency (570 mg doxorubicin per gram CTX-GO) via noncovalent interactions. Doxorubicin release was $\mathrm{pH}$-dependent and showed sustained-release properties. Cytotoxicity experiments demonstrated that CTX-GO/DOX mediated the highest rate of death of glioma cells compared with free doxorubicin or graphene oxide loaded with doxorubicin only. Further, conjugation with chlorotoxin enhanced accumulation of doxorubicin within glioma cells. These findings indicate that CTX-GO is a promising platform for drug delivery and provide a rationale for developing a glioma-specific drug delivery system.

Keywords: glioma, nanosheet, $\mathrm{pH}-$ dependent, cytotoxicity

\section{Introduction}

Graphene oxide exists as a two-dimensional nanosheet comprised of $\mathrm{sp}^{2}$ hybridized carbon atoms. The two-dimensional aromatic surface of graphene oxide makes it an ideal substrate for adsorption of antitumor drugs with aromatic rings by $\pi-\pi$ stacking interactions, resulting in high drug loading and a stable drug complex. ${ }^{1,2}$ In addition, its large specific surface area and abundant functional groups (epoxide, hydroxyl, and carboxylic groups) allow conjugation of further functional groups and synthesis of hybrid materials. ${ }^{3-5}$ Therefore, graphene oxide nanosheets have been increasingly investigated as a promising drug carrier for antitumor drug delivery.

Doxorubicin, a typical chemotherapy agent, can be loaded onto graphene oxide via $\pi-\pi$ stacking to investigate drug loading, controlled release, and drug delivery. Yang et $\mathrm{al}^{6}$ used graphene oxide as a nanocarrier to load doxorubicin noncovalently and investigated the release of doxorubicin in $\mathrm{pH}$-dependent manner. Recently, Zhang et $\mathrm{al}^{7}$ used graphene oxide nanosheets to load doxorubicin, and found that graphene oxide loaded with doxorubicin had sustained release behavior and antitumor efficacy.

Glioma is the most common and lethal primary brain tumor in humans. Treatment of glioma with chemotherapeutic drugs plays an important role in combination therapies. However, the outcome of chemotherapy is usually unsatisfactory due to poor drug accumulation at the tumor site and severe side effects. ${ }^{8-10}$ Despite great efforts to incorporate a doxorubicin-loaded graphene oxide (GO/DOX) complex into a drug delivery system, enhancement of drug accumulation at the tumor site is obviously limited using the enhanced permeability and retention effect alone. A well-known strategy for achieving 
efficient accumulation in tumor tissue is to conjugate drug carriers with specific ligands that can recognize molecular signatures on the cancer cell surface. ${ }^{11-13}$ Folic acid molecules have been covalently bound to graphene oxide along with antineoplastic drugs for specific targeting to MCF-7 cells overexpressing folate, and cellular uptake experiments indicate efficient and targeted delivery of anticancer drugs into cells via receptor-mediated endocytosis. ${ }^{14}$ More recently, Liu et $\mathrm{al}^{15}$ synthesized transferrin-modified graphene oxide and confirmed significantly improved therapeutic efficacy in glioma both in vitro and in vivo. Moreover, chlorotoxin, a 36-amino acid peptide isolated from the venom of the giant Israeli scorpion (Leiurus quinquestriatus), has attracted increasing attention due to its high selectivity for glioma and other tumors of neuroectodermal origin by binding to matrix metalloproteinase-2. ${ }^{16}$ Using chlorotoxin as a targeting ligand could overcome the shortcomings of currently widely used antibodies, which are bulky and show limited tissue penetration and cellular uptake when introduced in vivo. ${ }^{17}$ However, to date, chlorotoxin-conjugated graphene oxide (CTX-GO), which would be expected to have both high specificity and high drug loading efficiency for glioma-specific drug delivery, remains unexplored.

In this work, a glioma-targeted CTX-GO-based drug delivery system was developed (Figure 1). Doxorubicin, a chemotherapeutic agent used for treating a wide variety of cancers, ${ }^{18,19}$ was loaded onto CTX-GO in a noncovalent fashion. The specificity of CTX-GO and the therapeutic efficacy of doxorubicin loaded into CTX-GO was assayed in C6 glioma cells in vitro.

\section{Materials and methods Materials}

Carboxylated graphene oxide (GO-COOH) sheets were purchased from Nanjing XianFeng Nano Material Tech Co, Ltd (Nanjing, People's Republic of China). Fetal bovine serum and Dulbecco's Modified Eagle's Medium were acquired from Gibco (Basel, Switzerland). Cell Counting Kit-8 (CCK-8) was sourced from Dojindo Molecular Technologies, Inc., (Rockville, MD, USA). Doxorubicin hydrochloride was provided by Beijing Huafeng United Technology Co, Ltd (Beijing, People's Republic of China). Chlorotoxin (TM601) with a sequence of MCMPCFTTDHQMARKCDDCCGGKGRGKCYGPQCLCR was obtained from GL Biochem Ltd (Shanghai, People's Republic of China). N-hydroxysuccinimide was acquired from Acros Organics (Uppsala, Sweden). N-(3-dimethylaminopropyl)-N'- ethylcarbodiimide hydrochloride was provided by J\&K Chemical Ltd (Beijing, People's Republic of China). Other chemicals and reagents were of analytical grade and used as received.

\section{Conjugation of CTX-GO}

First, $150 \mathrm{mg}$ of carboxylated graphene oxide was dispersed in $20 \mathrm{~mL}$ of phosphate-buffered saline (0.1 M, pH 6.0),
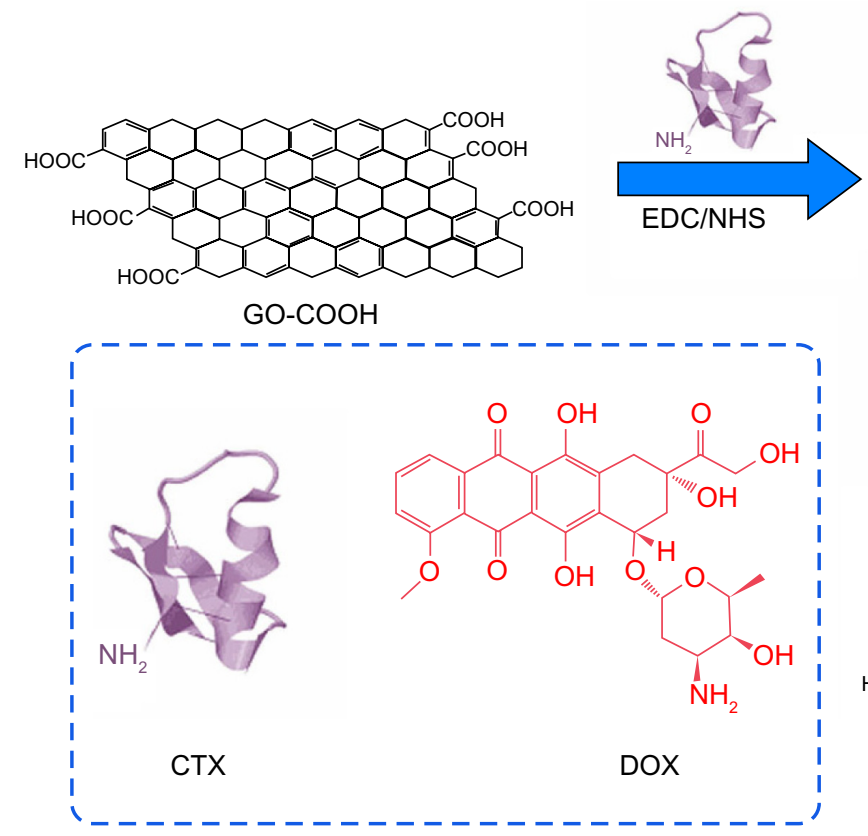
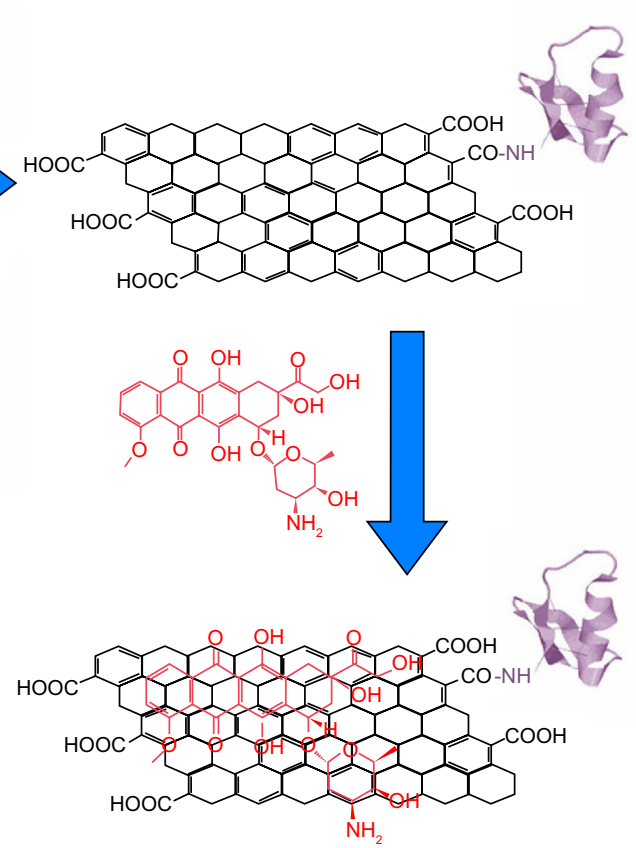

Figure I Schematic illustration of the preparation of CTX-GO/DOX.

Abbreviations: GO-COOH, carboxylated graphene oxide; DOX, doxorubicin; CTX, chlorotoxin; CTX-GO/DOX, chlorotoxin-conjugated graphene oxide loaded noncovalently with doxorubicin. 
followed by addition of $4 \mathrm{mg}$ of $\mathrm{N}$-hydroxysuccinimide and $6 \mathrm{mg}$ of $\mathrm{N}$-(3-dimethylaminopropyl)-N'-ethylcarbodiimide hydrochloride. After stirring for 30 minutes, the mixture was allowed to react with $15 \mathrm{mg}$ of chlorotoxin at room temperature for 48 hours in phosphate-buffered saline ( $\mathrm{pH}$ 8.0). The CTX-GO was then collected by centrifugation, washed three times with deionized water, and subjected to lyophilization.

\section{Loading of doxorubicin onto CTX-GO}

Loading of doxorubicin onto CTX-GO (CTX-GO/DOX) was done by simply mixing $1 \mathrm{mg}$ of CTX-GO with an appropriate amount of doxorubicin in $5 \mathrm{~mL}$ of water for 12 hours in the dark. The CTX-GO/DOX was then collected by centrifugation. To evaluate the loading efficiency of doxorubicin, ultraviolet-visible absorbance spectra for the initial doxorubicin solutions and supernatants were collected and the absorbance values at $485 \mathrm{~nm}$ were recorded. The amount of doxorubicin was then calculated according to the standard curve generated from a series of doxorubicin solutions with different concentrations.

\section{Effect of $\mathrm{pH}$ on release of doxorubicin}

To study the effect of $\mathrm{pH}$ on the release of doxorubicin from CTX-GO, $20 \mathrm{mg}$ of CTX-GO/DOX were dispersed in $2 \mathrm{~mL}$ of phosphate-buffered saline (10 mM, pH 7.4 and 5.0, respectively). The suspension was then placed in a dialysis bag with a molecular weight cutoff of $14 \mathrm{kDa}$. Subsequently, the dialysis bag was immersed in $20 \mathrm{~mL}$ of phosphatebuffered saline (10 mM, pH 7.4 and 5.0, respectively). At selected intervals, the buffered solution $(0.5 \mathrm{~mL})$ outside the dialysis bag was withdrawn and replaced with an equal volume of fresh-buffered solution. The amount of doxorubicin released was analyzed using the ultraviolet-visible spectrophotometer at $485 \mathrm{~nm}$.

\section{Characterizations}

Transmission electron microscopic images were obtained on a JEM-2100F system (JEOL, Tokyo, Japan) at an operating voltage of $200 \mathrm{kV}$. Specimens were prepared for transmission electron microscopic imaging by spreading a drop of sample on copper grids, followed by evaporation under vacuum. The topography and thickness of carboxylated graphene oxide was investigated using an atomic force microscope in contact mode. X-ray diffraction was carried out with a SmartLab X-ray diffractometer (Rigaku, Tokyo, Japan; $\mathrm{Cu}$ $\mathrm{K} \alpha$ radiation $\lambda=1.54056 \AA$ ) operating at $45 \mathrm{kV}$ and $200 \mathrm{~mA}$. Fourier transform infrared (FTIR) spectra were recorded on an Avatar 300 FTIR spectrometer (Thermo Nicolet Instrument Corporation, Waltham, MA, USA). Pristine graphene oxide or CTX-GO (2 mg) was mixed with $\mathrm{KBr}$ (98 $\mathrm{mg}$ ) and then pressed into a pellet with minimum pressure. One hundred scans were accumulated, with a resolution of $4 \mathrm{~cm}^{-1}$ for each spectrum. Ultraviolet-visible spectra were collected on a UV2550 ultraviolet-visible spectrophotometer (Shimadzu, Kyoto, Japan). The fluorescence spectra were recorded using an F-2500 fluorescence spectrophotometer (Hitachi, Tokyo, Japan) equipped with a $1 \mathrm{~cm}$ quartz cell (excitation $485 \mathrm{~nm}$, emission $585 \mathrm{~nm}$ ).

\section{Cell culture}

C6 (glioma) cells were routinely maintained in Dulbecco's Modified Eagle's Medium supplemented with 10\% fetal bovine serum, streptomycin $(100 \mu \mathrm{g} / \mathrm{mL})$, and penicillin $(100 \mathrm{U} / \mathrm{mL})$ at $37^{\circ} \mathrm{C}$ in a humidified $5 \% \mathrm{CO}_{2}$ atmosphere.

\section{In vitro cytotoxicity assay}

C6 cells were seeded into a 96-well plate at a density of $1 \times 10^{4}$ cells per well. After incubation for 24 hours, the cell culture medium was exchanged for fresh medium containing various doses of free doxorubicin, GO/DOX, and CTX-GO/ DOX, corresponding to equivalent doxorubicin concentrations of $1.0,2.5$, and $5.0 \mu \mathrm{g} / \mathrm{mL}$, respectively. Carboxylated graphene oxide and CTX-GO representing equivalent graphene oxide doses of $3.0,7.5$, and $15.0 \mu \mathrm{g} / \mathrm{mL}$, respectively, were used as controls. The cells were then allowed to incubate for a further 24 hours and the CCK- 8 assay was performed. Briefly, the cell culture medium was discarded and CCK-8 in fetal bovine serum-free Dulbecco's Modified Eagle's Medium $(0.1 \mathrm{mg} / \mathrm{mL})$ was added. After incubation for a further hour, the absorbance at $450 \mathrm{~nm}$ was measured using a Multiskan spectrum microplate reader (Thermo Electron Corporation, Waltham, MA, USA) and cell viability was calculated.

\section{Confocal laser scanning microscopy}

A total of $1 \times 10^{5} \mathrm{C} 6$ cells were reseeded on coverslips in each well of a 6-well plate and incubated for 24 hours. The growth medium was then replaced by doxorubicin, $\mathrm{GO} /$ DOX, or CTX-GO/DOX containing Dulbecco's Modified Eagle's Medium with an equivalent doxorubicin concentration of $0.5 \mu \mathrm{g} / \mathrm{mL}$. After incubation for a further 24 hours, the cells were rinsed three times with phosphate-buffered saline. The cells were then fixed with methanol for 10 minutes and dried slightly in air. For colocalization studies, the cells were stained with $0.5 \mu \mathrm{g} / \mathrm{mL}$ Hoechst 33258 for 10 minutes to stain the cell nucleus. The cells were washed with 
phosphate-buffered saline three times, and the coverslips were observed under a TCS SP5 confocal microscope (Leica Microsystems, Wetzlar, Germany). Hoechst 33258 was visualized at an excitation wavelength of $346 \mathrm{~nm}$, and doxorubicin was visualized on excitation at $511 \mathrm{~nm}$. High magnification images of the $\mathrm{C} 6$ cells were obtained using a $60 \times$ oil-immersion lens.

\section{Statistical analysis}

The data are shown as the mean \pm standard deviation. Differences were evaluated using the Student's $t$-test and considered to be statistically significant at $P<0.05$.

\section{Results and discussion}

\section{Characterizations}

The morphology of the carboxylated graphene oxide sheets was characterized by atomic force microscopy and transmission electron microscopy. As seen in Figure 2A, carboxylated graphene oxide had the flake-like shape of corrugated graphene sheets with a lateral width of $200 \mathrm{~nm}$. The crumpled structure was due to the scrolls and multiple folds of the flexible graphene oxide sheet. Note that each fold exhibited only one dark line, confirming that each exfoliated flake was a monolayer of graphene. The thickness of graphene oxide from the atomic force microscopy height profile was about $3 \mathrm{~nm}$ (Figure 2B), which is greater than that for a pure graphene oxide sheet as reported in the literature. ${ }^{20}$ This is probably due to the carboxyl groups on the basal plane of graphene oxide. ${ }^{21}$

The presence of carboxyl groups on the graphene oxide sheets was confirmed by FTIR (Figure 3A). The intensive band at $1,390 \mathrm{~cm}^{-1}$ was due to the symmetric stretching of -COO- from the basal plane of the graphene oxide sheets, whereas the band at $1,730 \mathrm{~cm}^{-1}$ originated from the asymmetric stretching of - $\mathrm{COOH}$ along the edges of the graphene oxide sheets. These bands diminished upon conjugation of chlorotoxin, and this was accompanied by appearance of amide I and II bands (Figure 3B), implying successful conjugation of chlorotoxin by the reaction of $\mathrm{NH}_{2}$ groups of chlorotoxin with the $\mathrm{COOH}$ groups of graphene oxide. The additional new peaks below $1,500 \mathrm{~cm}^{-1}$ observed in Figure 3B could be readily assigned to chlorotoxin by referring to the FTIR spectrum for free chlorotoxin (Figure 3C), further confirming the successful conjugation of chlorotoxin with the graphene oxide sheets.

The structural information for carboxylated graphene oxide and CTX-GO was assayed by X-ray diffraction, and the patterns obtained for carboxylated graphene oxide and CTX-GO are shown in Figure 4. It can clearly be seen that the graphene oxide sheets had a broad peak centered at $2 \theta=15.87^{\circ}$, corresponding to the (002) plane. This peak shifted downwards to $10.35^{\circ}$ and became sharper on conjugation of chlorotoxin, indicating partial reduction of graphene oxide under alkaline conditions during the chlorotoxin conjugation process. This, to some extent, confirmed successful conjugation of chlorotoxin onto the graphene oxide sheets.

To quantify the chlorotoxin conjugated onto the graphene oxide, the $\mathrm{C}, \mathrm{N}$ and $\mathrm{H}$ was content obtained by elemental analysis (Table 1). ${ }^{22}$ In comparison with carboxylated graphene oxide, CTX-GO showed a significant increase in $\mathrm{N}$ content from $0.101 \%$ to $2.095 \%$. Given that the $\mathrm{N}$ content in chlorotoxin is $18.219 \%$, the increased $\mathrm{N}$ content of CTX-GO
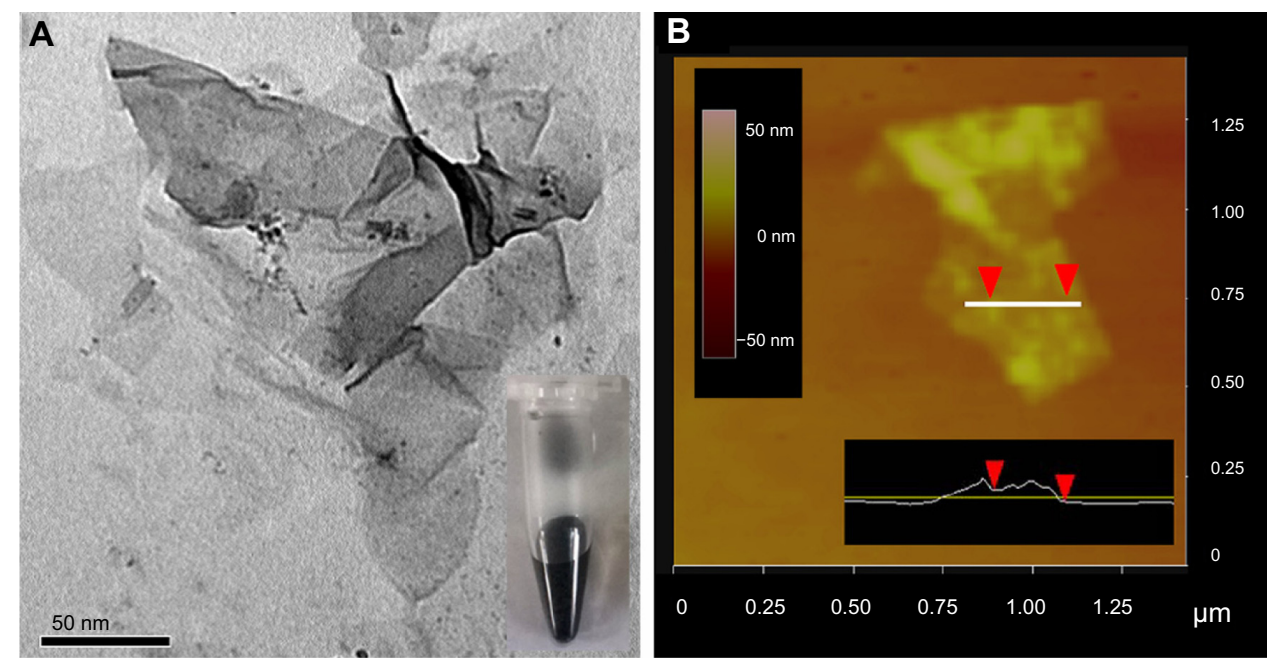

Figure 2 (A) Transmission electron microscopic images of carboxylated graphene oxide (inset: graphene dispersion in phosphate-buffered saline) and (B) atomic force microscopic images of carboxylated graphene oxide. 


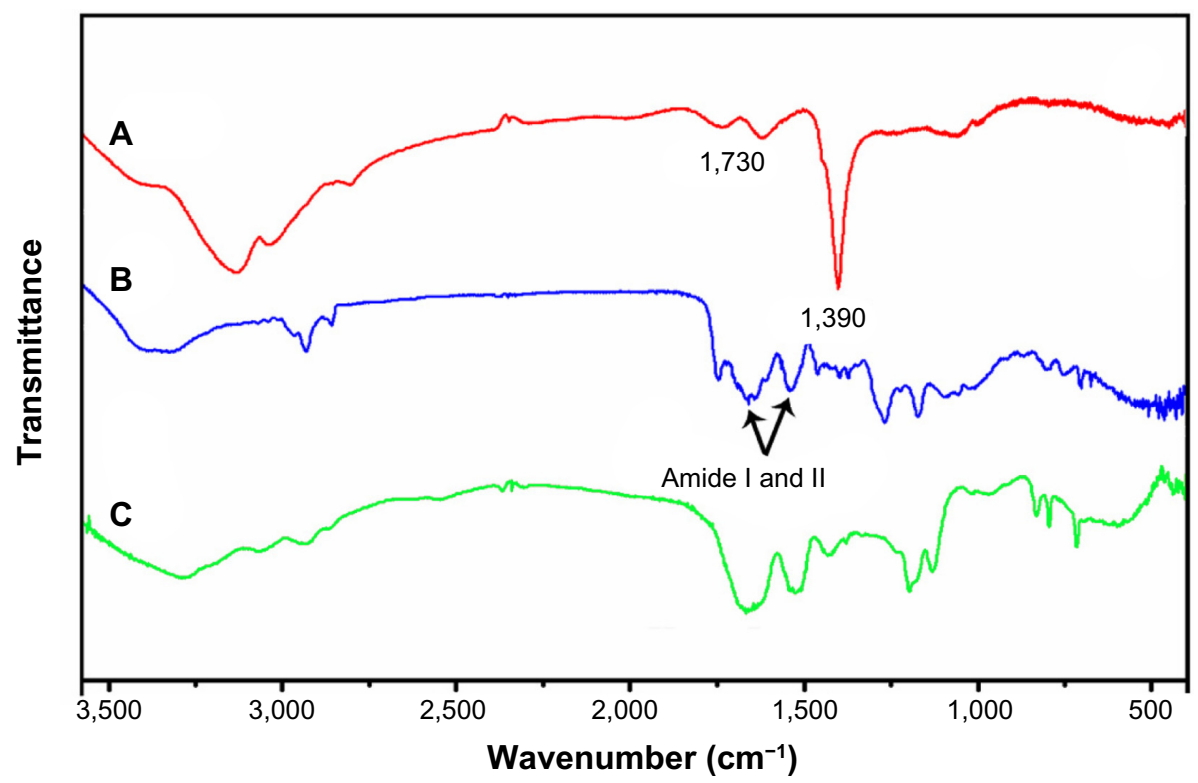

Figure 3 Fourier-transform infrared spectra of $(\mathbf{A})$ carboxylated graphene oxide, $(\mathbf{B})$ chlorotoxin-conjugated graphene oxide, and $(\mathbf{C})$ chlorotoxin.

indicates conjugation of chlorotoxin to graphene oxide. Further, based on the fact that the mass of $\mathrm{C}$ in CTX-GO was the sum of the $\mathrm{C}$ mass of chlorotoxin and CTX-GO, the amount of chlorotoxin conjugated to graphene oxide was estimated to be $1.88 \%$.

\section{Loading of doxorubicin onto CTX-GO}

Next, the CTX-GO sheets were loaded with doxorubicin. Graphene oxide is known to exist as a two-dimensional sheet consisting of $\mathrm{sp}^{2}$ hybridized carbon. The $\mathrm{sp}^{2}$ hybridized $\pi$ conjugated structure can thereby have $\pi$ - $\pi$ stacking interactions with the quinine portion of doxorubicin. In addition, the amine $\left(-\mathrm{NH}_{2}\right)$ and hydroxyl $(-\mathrm{OH})$ groups on doxorubicin can induce a strong hydrogen-bonding interaction with the $-\mathrm{COOH}$ and $-\mathrm{OH}$ groups on graphene oxide. Therefore, doxorubicin can be loaded onto CTX-GO in a noncovalent manner ${ }^{23}$ and the loading efficiency of doxorubicin was found to be around $570 \mathrm{mg}$ doxorubicin per gram CTX-GO.

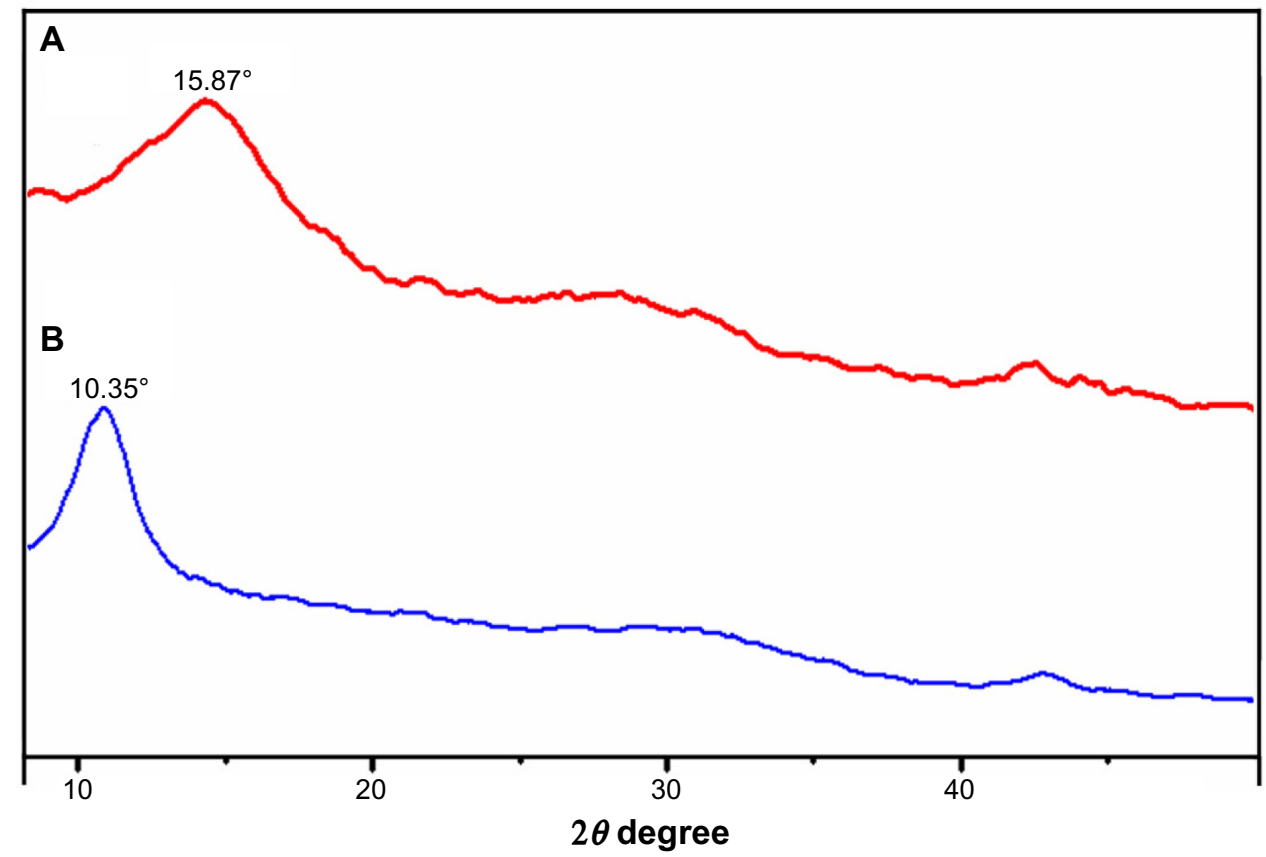

Figure 4 X-ray diffraction patterns of $(\mathbf{A})$ carboxylated graphene oxide and $(\mathbf{B})$ chlorotoxin-conjugated graphene oxide. 
Table I Elemental analysis results of GO-COOH and CTX-GO

\begin{tabular}{llll}
\hline & $\mathbf{C}$ & $\mathbf{H}$ & $\mathbf{N}$ \\
\hline GO-COOH & 28.55 & 2.530 & 0.101 \\
CTX-GO & 29.44 & 3.466 & 2.095
\end{tabular}

Abbreviations: $\mathrm{GO}-\mathrm{COOH}$, carboxylated graphene oxide; CTX-GO, chlorotoxinconjugated graphene oxide.

This value is higher than the loading efficiency reported for doxorubicin onto folate-conjugated graphene oxide. ${ }^{24}$

\section{Effect of $\mathrm{pH}$ on release of doxorubicin}

Release of doxorubicin from CTX-GO at $\mathrm{pH} 7.4$ (physiological $\mathrm{pH}$ ) and 5.0 (endosomal $\mathrm{pH}$ of cancer cells) was determined. The profiles for release of doxorubicin from chlorotoxin are shown in Figure 5. Release of doxorubicin from CTX-GO was clearly pH-dependent. Specifically, doxorubicin was released in a slower and controlled manner from CTX-GO at $\mathrm{pH}$ 7.4. A possible explanation for this phenomenon is that the hydrogen-bonding interaction between doxorubicin and graphene oxide is less significant in acidic conditions. Under acidic conditions, protonation of the amine $\left(-\mathrm{NH}_{2}\right)$ groups in doxorubicin results in partial dissociation of the hydrogen-bonding interaction, hence the amount of doxorubicin released from CTX-GO was greater. However, the percentage of unreleased doxorubicin on CTX-GO sheets after 60 hours was still more than $70 \%$, even under acidic conditions, confirming the presence of strong $\pi-\pi$ stacking interactions between CTX-GO and doxorubicin in addition to hydrogen bonding. Nevertheless, the $\mathrm{pH}$-dependent release of doxorubicin from CTX-GO is valuable for drug delivery, given that the microenvironments in the extracellular tissue around tumors and intracellular lysosomes and endosomes are acidic. $^{25}$

\section{In vitro cytotoxicity assay}

To evaluate specific targeting capacity, the in vitro cytotoxicity of GO/DOX and CTX-GO/DOX was compared using the CCK-8 assay. Carboxylated graphene oxide, CTX-GO, and free doxorubicin were used as negative and positive controls, respectively. As shown in Figure 6, both carboxylated graphene oxide and CTX-GO had negligible toxic effects on C6 cells (viability was $>80 \%$ at all concentrations). However, the difference in cytotoxicity between carboxylated graphene oxide and CTX-GO became more distinct with increasing graphene oxide concentration, implying more cellular uptake of CTX-GO by C6 cells due to the ability of chlorotoxin to target $\mathrm{C} 6$ cells. As expected, doxorubicin loaded into either GO/DOX or CTX-GO/DOX had enhanced cytotoxicity compared with free doxorubicin. However, CTX-GO/DOX showed greater cytotoxicity at $2.5 \mu \mathrm{g} / \mathrm{mL}$ and $5 \mu \mathrm{g} / \mathrm{mL}$. Clearly, the enhancement of inhibition is attributed to the targeting specificity of chlorotoxin, which leads to greater uptake of doxorubicin by C6 cells.

To provide additional evidence of the differential cytotoxicity of GO/DOX and CTX-GO/DOX in C6 cells, the morphologies of these cells were observed after incubation with medium alone (control), carboxylated graphene oxide, CTX-GO, GO/DOX, CTX-GO/DOX, and free doxorubicin for

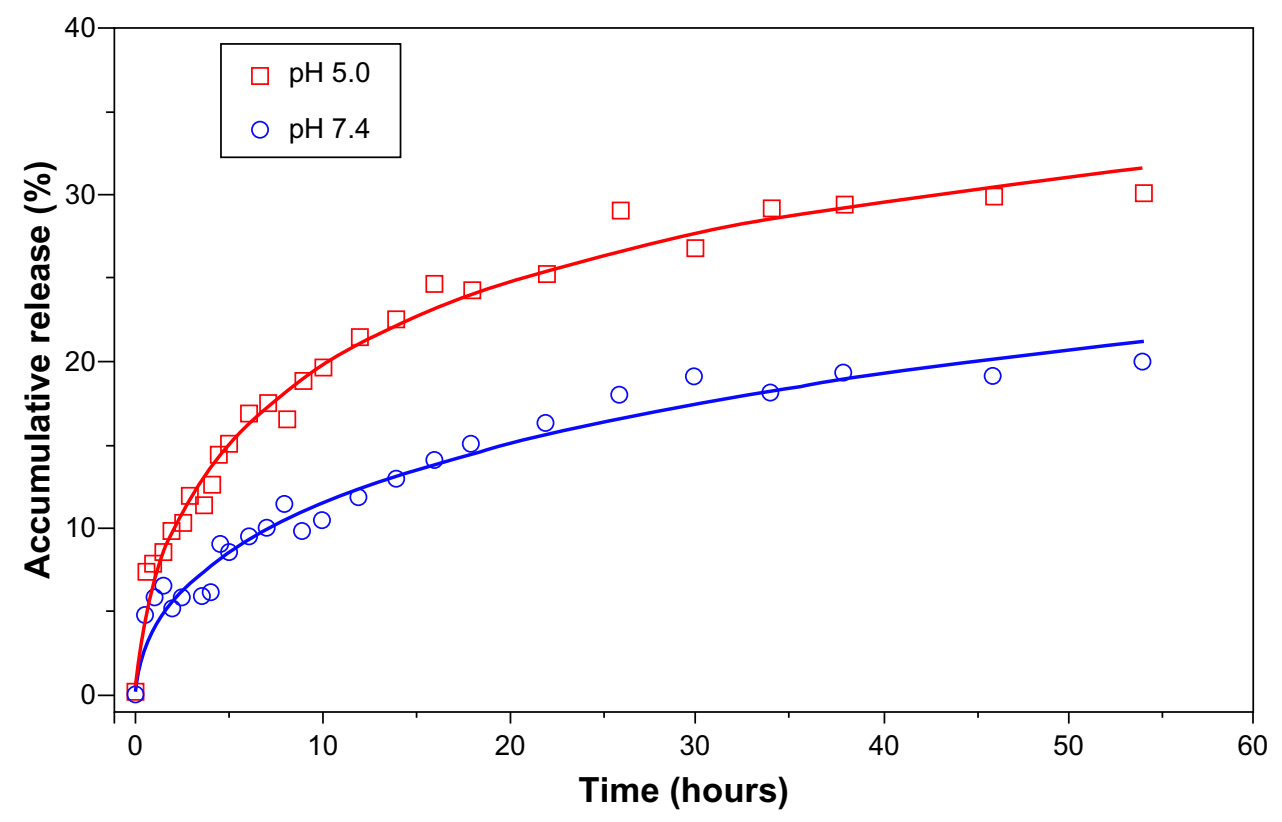

Figure 5 Release profiles of doxorubicin from chlorotoxin-conjugated graphene oxide at pH 7.4 and pH 5.0. 


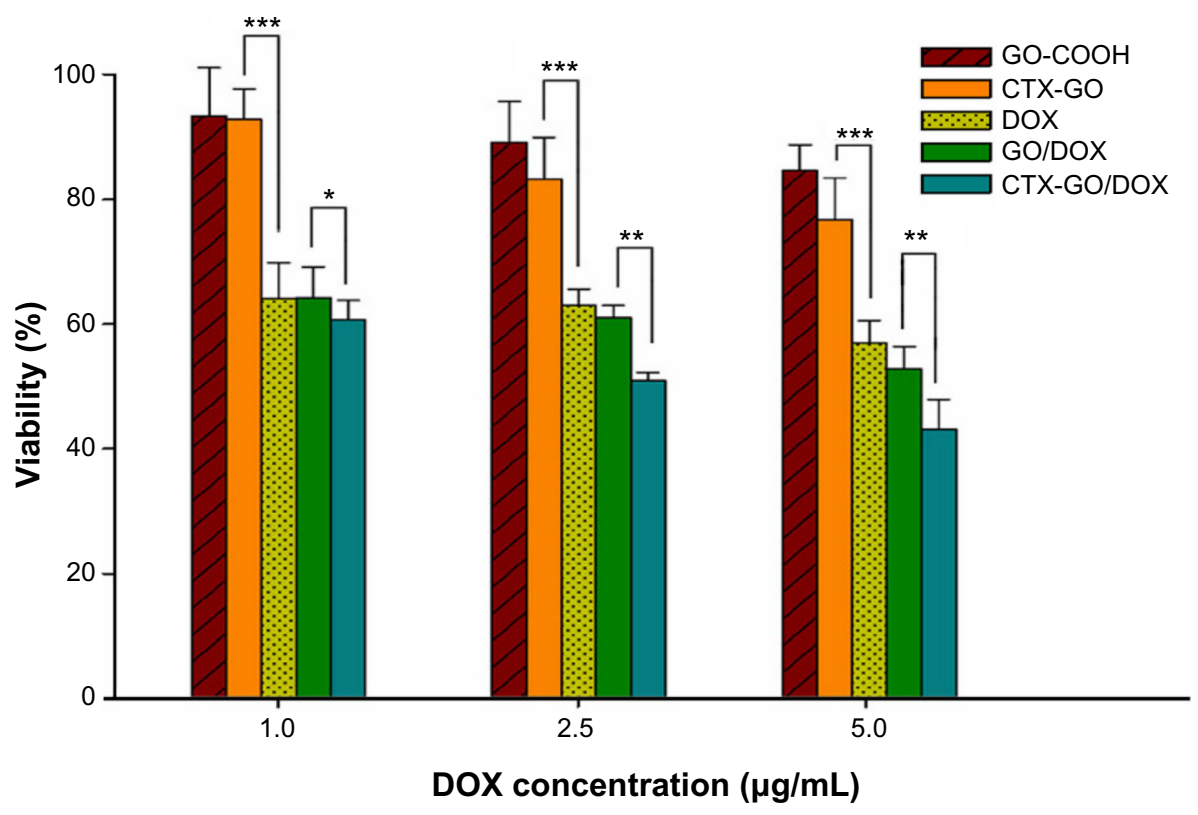

Figure 6 Viability of C6 glioma cells determined by Cell Counting Kit-8 assay after 24 hours of incubation with GO-COOH, CTX-GO, DOX, GO/DOX, and CTX-GO/DOX at various concentrations. Each data represents the mean \pm standard deviation of three experiments. Statistically significant differences were evaluated using the Student's $t$-test $(* P<0.05, * * P<0.01$, $* * * P<0.001)$.

Abbreviations: GO-COOH, carboxylated graphene oxide; CTX-GO, chlorotoxin-conjugated graphene oxide; DOX, doxorubicin; GO/DOX, graphene oxide loaded noncovalently with doxorubicin; CTX-GO/DOX, chlorotoxin-conjugated graphene oxide loaded noncovalently with doxorubicin.

24 hours using optical microscopy. As shown in Figure 7, no significant changes in cell density or morphology were found for graphene oxide or CTX-GO when compared with the control group, suggesting biocompatibility of carboxylated graphene oxide. In contrast, treatment with GO/DOX, CTX-GO/
DOX, or free doxorubicin (at an equivalent doxorubicin concentration of $2.5 \mu \mathrm{g} / \mathrm{mL}$ ) resulted in significant morphological changes, including cell detachment from the plate, shrinking, and appearance of spherical cells. Moreover, CTX-GO/DOX induced more pronounced cell morphological changes and
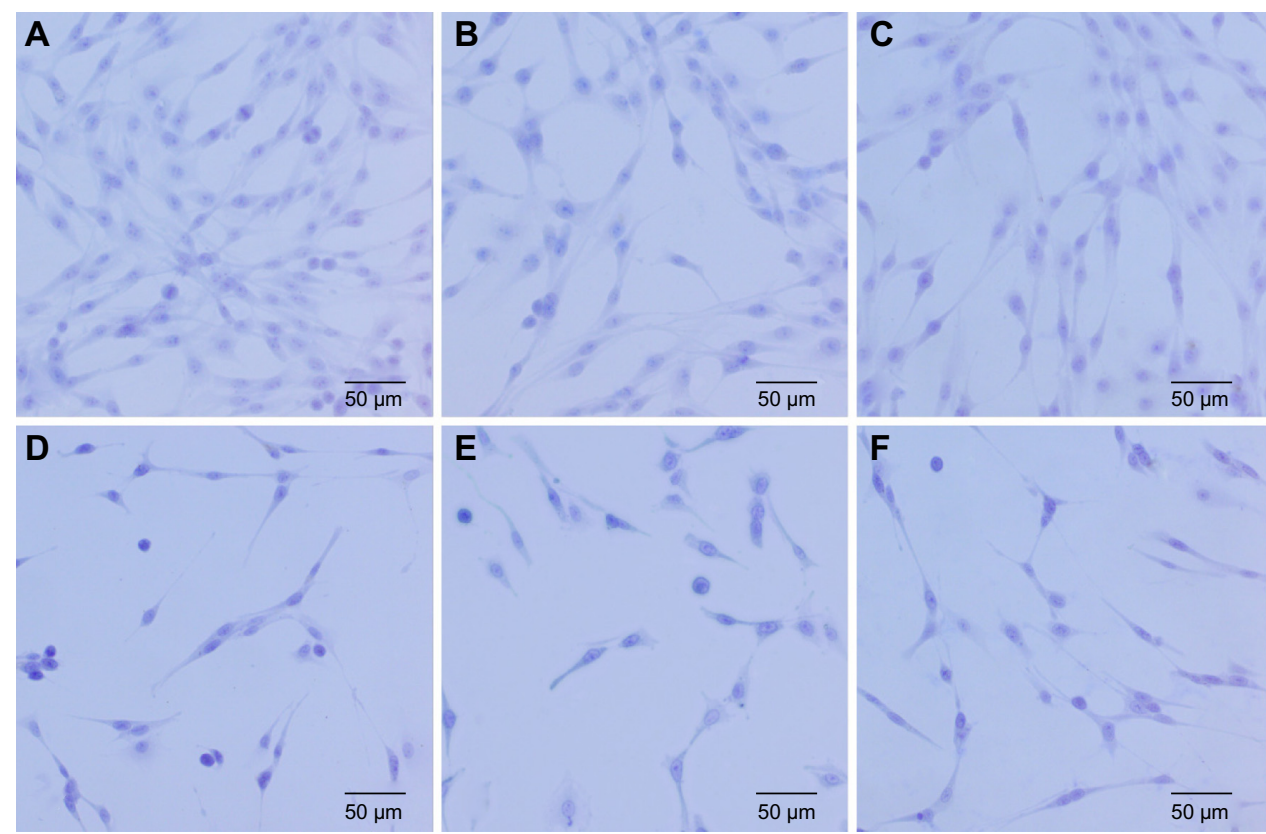

Figure 7 Microscopic images of C6 cells treated with (A) medium (control), (B) carboxylated graphene oxide, (C) chlorotoxin-conjugated graphene oxide, and (D) graphene oxide loaded noncovalently with doxorubicin, (E) chlorotoxin-conjugated graphene oxide loaded noncovalently with doxorubicin, and (F) free doxorubicin at an equivalent doxorubicin concentration of $2.5 \mu \mathrm{g} / \mathrm{mL}$ for 24 hours. 


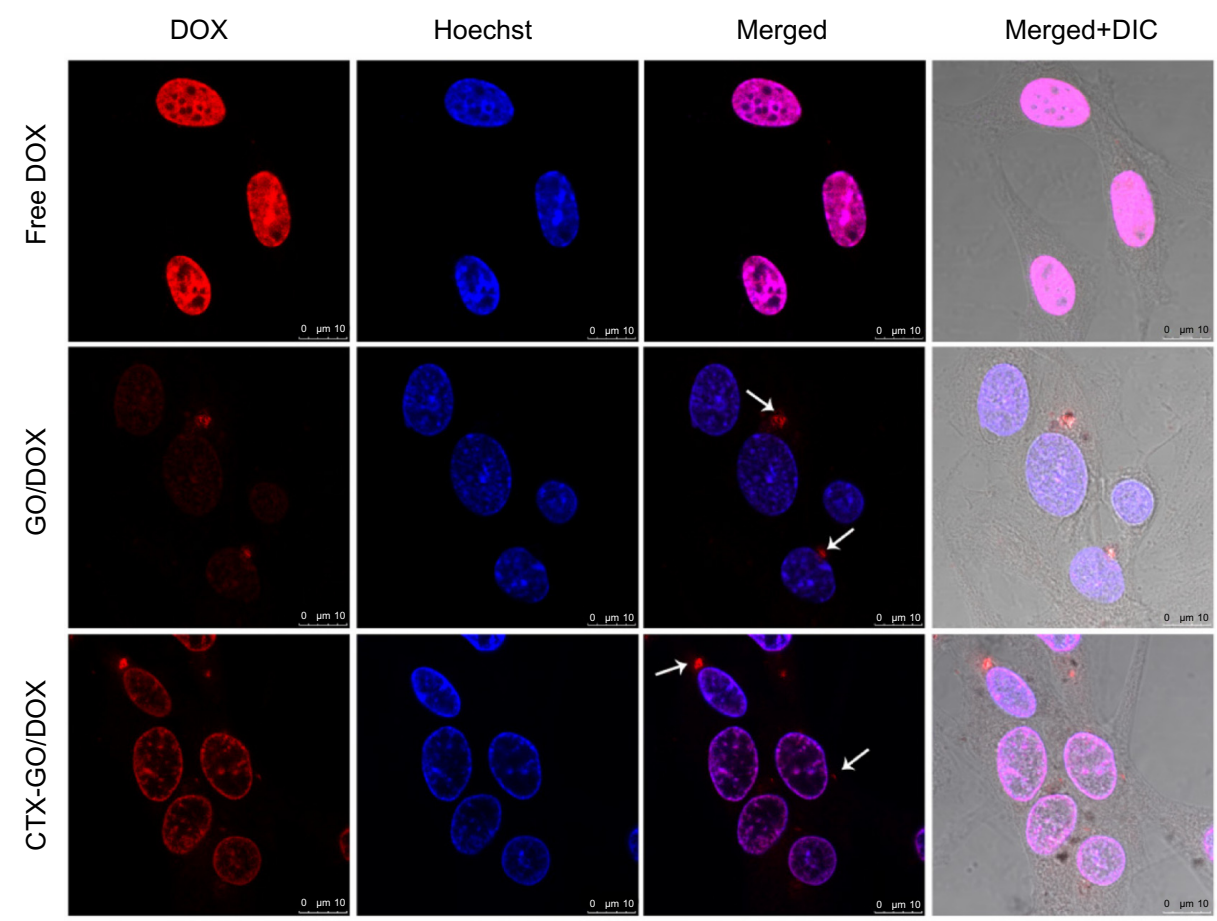

Figure 8 Cellular localization and distribution of DOX, GO/DOX, and CTX-GO/DOX in C6 cells with the equivalent concentration of DOX (0.5 $\mu$ g/mL) for 24 hours of incubation. The nuclei were stained with Hoechst 33258. Scale bar $10 \mu \mathrm{m}$.

Note: The red dots the arrows are pointing to are GO/DOX or CTX-GO/DOX

Abbreviations: DIC, differential interference contrast; DOX, doxorubicin; GO/DOX, graphene oxide loaded noncovalently with doxorubicin; CTX-GO/DOX, chlorotoxin conjugated graphene oxide loaded noncovalently with doxorubicin.

cell death than GO/DOX or free doxorubicin, indicating the targeting capability of chlorotoxin. These results correlate well with the data obtained from the CCK-8 assay.

\section{Confocal laser scanning microscopy}

The uptaking process (Figure S1, Supplementary material) and the intracellular localization of doxorubicin, $\mathrm{GO} /$ DOX, and CTX-GO/DOX in C6 cells was investigated by confocal laser scanning microscopy. As shown in Figure 8, doxorubicin, GO/DOX, and CTX-GO/DOX showed different distribution patterns in C6 cells. Treatment with free doxorubicin produced a bright purple color inside the cell nuclei, suggesting that free doxorubicin was primarily distributed in the nuclei. In contrast, treatment with $\mathrm{GO} /$ DOX and CTX-GO/DOX produced a relatively weak fluorescence signal in the cell nuclei and aggregated red dots in the cytoplasm (on differential interference contrast), indicating that only small amounts of doxorubicin were released and entered the cell nuclei. However, compared with GO/DOX, a greater purple fluorescence signal in both the nuclei and cytoplasm were observed for CTX-GO/ DOX, confirming that more doxorubicin is delivered into the cell upon conjugation with chlorotoxin. This suggests a chlorotoxin-specific interaction with the receptor distributed on the cell membrane, leading to more accumulation of doxorubicin in C6 cells.

\section{Conclusion}

In summary, CTX-GO sheets were prepared for gliomatargeted delivery of the anticancer drug doxorubicin. It was demonstrated that doxorubicin molecules could be efficiently loaded onto CTX-DOX via noncovalent interactions and that the release of doxorubicin from CTX-GO is pH-dependent. The cytotoxicity study demonstrated that CTX-GO/DOX induced the highest rate of glioma cell death compared with GO/DOX and free doxorubicin, probably due to the fact that conjugation with chlorotoxin enhanced the accumulation of doxorubicin within the cells. Our findings suggest that CTX-GO is a potential drug delivery platform and provide a rationale for developing glioma-specific targeted delivery of an antitumor drug. Further in vivo study is still needed and obstacles, such as the blood-brain barrier should not be overlooked.

\section{Acknowledgments}

The authors are grateful for the financial support of the Natural Science Foundation of China (81271388, 81271639, 81070977, and 81200931), the Scientific Research Common Program of Beijing Municipal Commission of 
Education (KM20110025007), and the Beijing Municipal Foundation for Talents (2011D005018000001).

\section{Author contributions}

QX carried out the mechanism analysis and drafted the manuscript. HW performed the biological part of the experimental work, and participated in the design of the study and in drafting of the manuscript. WG participated in the experimental work, carried out the characterizations, and contributed to the design of the study and drafting of the manuscript. NX participated in the experimental work and carried out the FTIR. LY participated in the design of the study and helped draft the manuscript. All authors read and approved the final paper.

\section{Disclosure}

The authors report no conflicts of interest in this work.

\section{References}

1. Sahu A, Choi WI, Lee JH, et al. Graphene oxide mediated delivery of methylene blue for combined photodynamic and photothermal therapy. Biomaterials. 2013;34(26):6239-6248.

2. Shen H, Zhang L, Liu M, et al. Biomedical applications of graphene. Theranostics. 2012;2(3):283-294.

3. Yang Y, Zhang YM, Chen Y, et al. Construction of a graphene oxide based noncovalent multiple nanosupramolecular assembly as a scaffold for drug delivery. Chemistry. 2012;18(14):4208-4215.

4. Qin XC, Guo ZY, Liu ZM, et al. Folic acid-conjugated graphene oxide for cancer targeted chemo-photothermal therapy. J Photochem Photobiol B. 2013;120:156-162.

5. Zhang Y, Nayak TR, Hong H, et al. Graphene: a versatile nanoplatform for biomedical applications. Nanoscale. 2012;4(13):3833-3842.

6. Yang XY, Zhang XY, Liu ZF, et al. High-efficiency loading and controlled release of doxorubicin hydrochloride on graphene oxide. $J$ Phys Chem C. 2008;112(45):17554-17558.

7. Zhang LM, Xia JG, Zhao QH, et al. Functional graphene oxide as a nanocarrier for controlled loading and targeted delivery of mixed anticancer drugs. Small. 2010;6(4):537-544.

8. Steichen SD, Caldorera-Moore M, Peppas NA. A review of current nanoparticle and targeting moieties for the delivery of cancer therapeutics. Eur J Pharm Sci. 2012;48(3):416-427.

9. Raudino G, Caffo M, Caruso G, et al. Nanoparticle-based cerebral drugdelivery systems and antiangiogenic approach in gliomas treatment Recent Pat Nanotechnol. 2011;5(3):239-244.
10. Yang XY, Wang YS, Huang X, et al. Multi-functionalized graphene oxide based anticancer drug-carrier with dual-targeting function and pH-sensitivity. J Mater Chem. 2011;21(10):3448-3454.

11. Panzarini E, Inguscio V, Tenuzzo BA, et al. Nanomaterials and autophagy: new insights in cancer treatment. Cancers (Basel). 2013;5:296-319.

12. Kang MJ, Park SH, Kang MH, et al. Folic acid-tethered Pep-1 peptideconjugated liposomal nanocarrier for enhanced intracellular drug delivery to cancer cells: conformational characterization and in vitro cellular uptake evaluation. International Journal of Nanomedicine. 2013;8:1155-1165.

13. Shi S, Yang K, Hong $\mathrm{H}$, et al. Tumor vasculature targeting and imaging in living mice with reduced graphene oxide. Biomaterials. 2013;34(12):3002-3009.

14. Huang P, Xu C, Lin J, et al. Folic acid-conjugated graphene oxide loaded with photosensitizers for targeting photodynamic therapy. Theranostics. 2011;1:240-250

15. Liu G, Shen H, Mao J, et al. Transferrin modified graphene oxide for glioma-targeted drug delivery: in vitro and in vivo evaluations. $A C S$ Appl Mater Interfaces. 2013;5(15):6909-6914.

16. Sun C, Veiseh O, Gunn J, et al. In vivo MRI detection of gliomas by chlorotoxin-conjugated superparamagnetic nanoprobes. Small. 2008;4(3):372-379.

17. Huang R, Ke W, Han L, et al. Targeted delivery of chlorotoxin-modified DNA-loaded nanoparticles to glioma via intravenous administration. Biomaterials. 2011;32(9):2399-2406.

18. Arap W, Pasqualini R, Ruoslahti E. Cancer treatment by targeted drug delivery to tumor vasculature in a mouse model. Science. 1998;279(5349):377-380.

19. Cutts SM, Nudelman A, Rephaeli A, et al. The power and potential of doxorubicin-DNA adducts. IUBMB Life. 2005;57(2):73-81.

20. McAllister MJ, Li JL, Adamson DH, et al. Single sheet functionalized graphene by oxidation and thermal expansion of graphite. Chem Mater. 2007;19(18):4396-4404.

21. Stankovich S, Dikin DA, Piner RD, et al. Synthesis of graphene-based nanosheets via chemical reduction of exfoliated graphite oxide. Carbon. 2007;45(7):1558-1565.

22. Ying Q, Liang S, Xixuan L, et al. Highly water-dispersible TiO2 nanoparticles for doxorubicin delivery: effect of loading mode on therapeutic efficacy. J Mater Chem. 2011;21:18003-18010.

23. Ali-Boucetta H, Al-Jamal KT, McCarthy D, et al. Multiwalled carbon nanotube-doxorubicin supramolecular complexes for cancer therapeutics. Chem Commun. 2008;(4):459-461.

24. Wei G, Yan M, Dong R, et al. Covalent modification of reduced graphene oxide by means of diazonium chemistry and use as a drug-delivery system. Chemistry. 2012;18(46):14708-14716.

25. Hilder TA, Hill JM. Modeling the loading and unloading of drugs into nanotubes. Small. 2009;5(3):300-308. 


\section{Supplementary material}

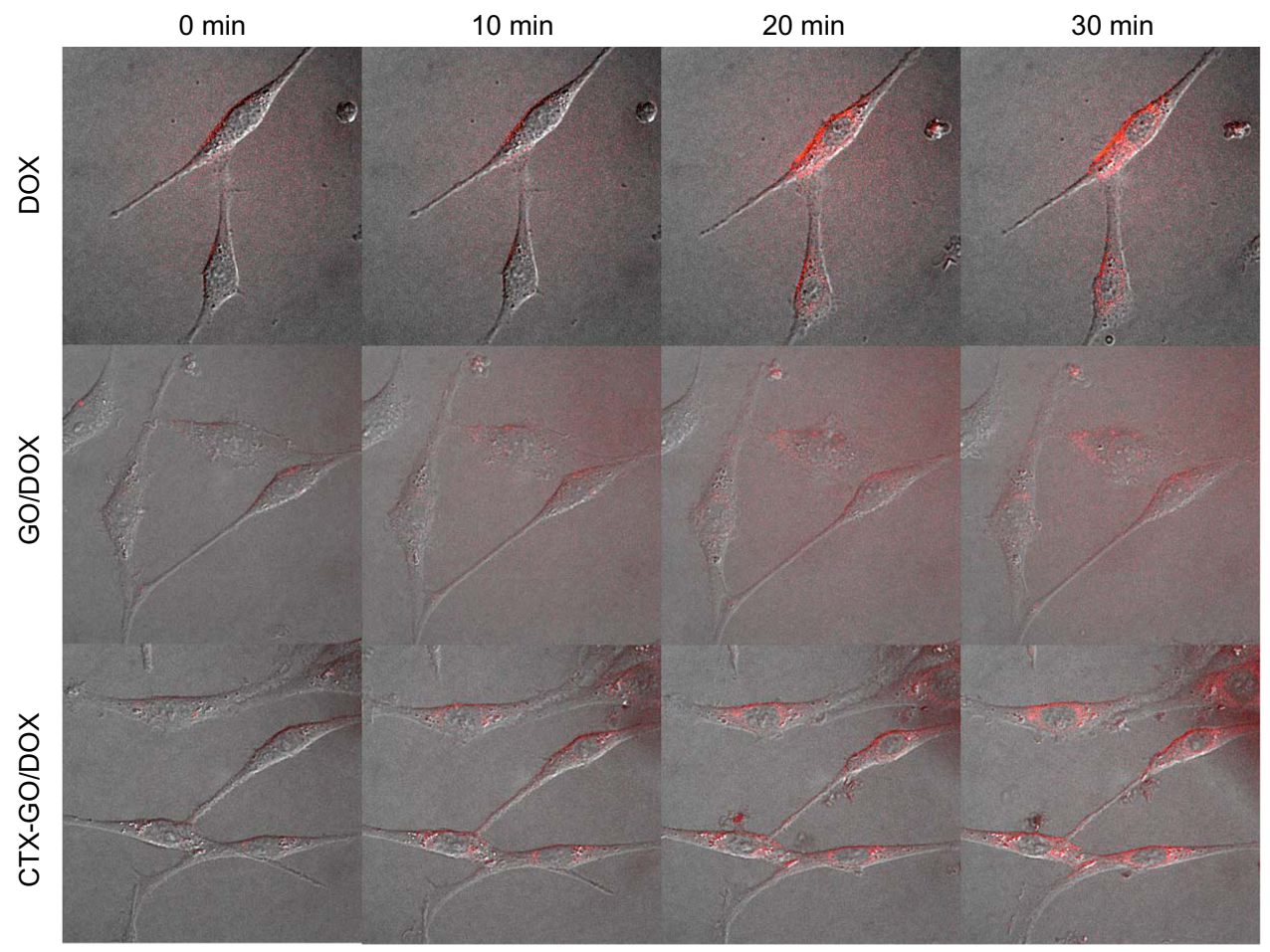

Figure SI Intracellular localization following 0, 10, 20, 30 minutes of coincubation time in C6 cells with free DOX, GO/DOX, or CTX-GO/DOX with the equivalent concentration of DOX $(5.0 \mu \mathrm{g} / \mathrm{mL})$. The cells were imaged on a Olympic microscope with an UltraVIEW Vox confocal attachment (Perkin Elmer, Boston, MA, USA). Laser lines were used at $488 \mathrm{~nm}$ and $560 \mathrm{~nm}$.

Abbreviations: DOX, doxorubicin; GO/DOX, graphene oxide loaded noncovalently with doxorubicin; CTX-GO/DOX, chlorotoxin-conjugated graphene oxide loaded noncovalently with doxorubicin; min, minutes.

\section{Publish your work in this journal}

The International Journal of Nanomedicine is an international, peerreviewed journal focusing on the application of nanotechnology in diagnostics, therapeutics, and drug delivery systems throughout the biomedical field. This journal is indexed on PubMed Central, MedLine, CAS, SciSearch $\AA$, Current Contents ${ }^{\circledR} /$ Clinical Medicine,
Journal Citation Reports/Science Edition, EMBase, Scopus and the Elsevier Bibliographic databases. The manuscript management system is completely online and includes a very quick and fair peer-review system, which is all easy to use. Visit http://www.dovepress.com/ testimonials.php to read real quotes from published authors. 